\title{
The karyotype of Aegilops geniculata and its use to identify both addition and substitution lines of wheat
}

\author{
Yingjin $\mathrm{Yi}^{\dagger}$, Ke Zheng ${ }^{\dagger}$, Shunzong Ning ${ }^{\dagger}$, Laibin Zhao, Kai Xu, Ming Hao, Lianquan Zhang, Zhongwei Yuan and \\ Dengcai Liu* (10
}

\begin{abstract}
Background: The annual allotetraploid species Aegilops geniculata harbors a number of traits relevant for wheat improvement. An effective cytogenetic method has yet to be developed to distinguish between each of its 14 chromosomes.

Results: A fluorescence in situ hybridization (FISH) approach was adopted to describe the karyotype of Ae. geniculata. Each of its 14 chromosomes was unequivocally recognized using a cocktail of three probes, namely pTa-713, (AAC) 5 and pTa71. FISH karyotyping was then used to detect and characterize selections from an Ae. geniculata $\times$ bread wheat wide cross of a chromosome $1 \mathrm{M}^{9}$ disomic addition line and three $4 \mathrm{M}^{9}(4 \mathrm{~B})$ substitution lines. The identity of the addition line was confirmed by the presence of Glu-M1, detected both using an SDS-PAGE separation of endosperm proteins and by applying a PCR assay directed at the Glu-M1 locus. The status of the substitution lines was validated by genotyping using a wheat single nucleotide polymorphism chip.

Conclusion: FISH karyotyping based on pTa-713, (AAC) $)_{5}$ and pTa71 will be useful for determining the contribution of Ae. geniculata to derivatives of an Ae. geniculata $\times$ wheat wide cross. SNP chip-based genotyping is effective for confirming the status of whole chromosome wheat/alien substitution lines.
\end{abstract}

Keywords: Substitution line, Addition line, Fluorescence in situ hybridization, Single nucleotide polymorphism

\section{Background}

Aegilops geniculata Roth (syn. Ae. ovata L, $2 \mathrm{n}=4 \mathrm{x}=28$, genome formula $\mathrm{U}^{\mathrm{g}} \mathrm{M}^{\mathrm{g}}$ ) represents a source of potentially useful genetic variation of relevance to bread wheat improvement. The species is thought to represent a natural allotetraploid between the diploid Ae. umbellulata Zhuk. (U genome carrier) and Ae. comosa Sibth. et Sm. (M genome carrier) [1]. A number of genes conferring resistance to various diseases have been transferred from this species into bread wheat, notably $\mathrm{Yr} 40$ (resistance against stripe rust), $\operatorname{Lr} 57$ (leaf rust) [2], Sr53 (stem rust) [3] and Pm29 (powdery mildew) [4, 5]. Some accessions of Ae. geniculata have displayed high levels of water use efficiency [6], and the species overall exhibit a higher

\footnotetext{
* Correspondence: dcliu7@sicau.edu.cn

${ }^{\dagger}$ Yingjin Yi, Ke Zheng and Shunzong Ning contributed equally to this work. Triticeae Research Institute, Sichuan Agricultural University, Chengdu 611130, Sichuan, China
}

tolerance to moisture stress than any of the related species Ae. markgrafii (Greuter) K. Hammer, Ae. longissima Schweinf. \& Muschl., Ae. searsii Feldman et Kislev ex K. Hammer or Ae. speltoides Tausch [7]. It also carries alleles at the genes encoding endosperm proteins which have been predicted to improve the end-use quality of bread wheat $[8,9]$.

Genomic in situ hybridization (GISH) has been a very successful technique for discriminating between the chromosomes belonging to the various genomes represented in Triticeae species, while fluorescence in situ hybridization (FISH) tends to be used for identifying individual chromosomes. A FISH-based karyotype of Ae. geniculata has been established, employing the probe combination pSc119.2, Afa family repeats, pAs1 and pTa71 [10-12]. However, some segments of the Ae. geniculata genome lack any probe hybridization sites, meaning that FISH karyotyping needs to be supported 
for the identification of non-intact chromosomes by a GISH-based analysis. For example, the sites of pSc119.2 hybridization are concentrated close to the telomeres of most chromosome arms, making it difficult to differentiate between chromosomes $1 \mathrm{U}^{\mathrm{g}}, 2 \mathrm{U}^{\mathrm{g}}, 3 \mathrm{M}^{\mathrm{g}}$, and $4 M^{g}[10,12]$. Here, the objective was to develop a FISH assay able to unequivocally recognize each of the 14 Ae. geniculata chromosomes, and to use this assay to characterize a number of derivatives of an Ae. geniculata $\times$ wheat wide cross.

\section{Methods}

\section{Plant materials}

The following taxa were used in these experiments: Ae. umbellulata ( $2 \mathrm{n}=2 \mathrm{x}=14$, carrier of the U genome) accession AS4, Ae. comosa $(2 \mathrm{n}=2 \mathrm{x}=14$, carrier of the $\mathrm{M}$ genome) accession PI551068, Ae. geniculata accession AS6, the bread wheat cultivars Yi-yuan 2 (YY2), Chinese Spring and Chuan-mai 41 (CM41), and ten $\mathrm{F}_{7}$ derivatives of a wide cross between Ae. geniculata and wheat (pedigree AS6/YY2//YY2/3/CM41).

\section{GISH and FISH analysis}

Cytological preparations were carried out using the methods described by Zhao et al. [13]. For GISH analyses, total genomic DNA was extracted from fresh leaves of Ae. comosa and Ae. geniculata, and labeled with digoxigenin-11-dUTP (Roche Diagnostics GmbH, Mannheim, Germany) via nick translation to use as the probe; non-labeled total genomic DNA of Ae. umbellulata and CM41 were used for blocking. The GISH procedure was based on the protocol described by Hao et al. [14], with the exception that the concentration of the probe DNA was changed to $0.1 \mu \mathrm{g} / \mu \mathrm{L}$ and that of blocking DNA to $3.5 \mu \mathrm{g} / \mu \mathrm{L}$. FISH experiments were conducted based on the methods given by Zhao et al. [15]. The following probes were essayed: Afa family repeats [16], pSc119.2, pTa-535 and pTa71 [17], (AAC) $)_{5}$ [18], $(\mathrm{CTT})_{5}$ and pTa-713 [13]. Probes were labeled with either FAM or TAMRA by the TsingKe Biological Technology Company (Chengdu, China). The preparations were stained with DAPI (Vector Laboratories Inc., Burlingame, CA, USA) and the fluorescence signals visualized and captured using an BX-63 epifluorescence microscope equipped with a Photometric SenSys DP70 CCD camera (Olympus, Tokyo, Japan). Raw images were processed using Photoshop v.7.1 (Adobe Systems Inc., San Jose, CA, USA).

\section{Single nucleotide polymorphism (SNP) genotyping}

Genomic DNA was extracted from fresh leaves using a plant genomic DNA kit (Tiangen Biotech, Beijing Co. Ltd., Beijing, China). Chip-based genotyping was carried out using the CapitalBio Wheat $55 \mathrm{~K}$ SNP array. (www.capitalbio.com); the SNP loci arrayed on this chip represent a sub-set of the Affymetrix ${ }^{\ominus}$ Axiom $^{\ominus}$ Wheat 660 chip, as selected by the Institute of Crop Science, Chinese Academy of Agricultural Sciences (wheat.pw. usda.gov/ggpages/topics/Wheat660_SNP_array_developed_by_CAAS.pdf). The flanking sequences of each locus were used to map each site onto the bread wheat reference sequence (urgi.versailles.inra.fr/download/ iwgsc/IWGSC_RefSeq_Assemblies/v1.0/), by imposing a BLASTN E-value threshold of $10^{-10}$, allowing a maximum mismatch of one base. For lines concluded to harbor an Ae. geniculata chromosome substituting for a wheat chromosome, the ratio between the observed and expected number of markers on the wheat chromosome in question (4B) was calculated by considering a series of $10 \mathrm{Mb}$ intervals along the chromosome, applying a sliding window of $10 \mathrm{Mb}$ and a step length of 1 $\mathrm{Mb}$. A graphical representation of these ratios was obtained using the R package ggplot2 v.2.2.1 [19]

\section{High molecular weight glutenin subunit (HMW-GS) analysis}

The extraction of protein from single grains and their separation using sodium dodecyl sulfate polyacrylamide gel electrophoresis (SDS-PAGE) followed methods given by Yan et al. [20]. After electrophoresis for $150 \mathrm{~min}$ (120 V, 20 $\mathrm{mA}$ ), the gels were stained with Coomassie Brilliant Blue $\mathrm{R}-250$ staining solution for $1 \mathrm{~h}$, then destained in distilled water. The primer pair $5^{\prime}$-ATGGCTAAGCGGYTRGTCC TCTTTG/5'-CTATCACTGGCTRGCGGACAATGG was used to amplify the coding region of the whole set of Glu-1 genes [21]. The methods required for PCR amplification, cloning and sequencing followed those given by Guo et al. [21].

\section{Development of a PCR assay for Glu-M1x}

In a search for informative SNPs, a multiple sequence alignment was carried out of the gene sequences encoding the HMW-GS 1Dx2 (Genbank accession KF466259.1), 1Ax (JQ007589.1), 1Bx13 (EF540764.1), 1Ux (KX375406.1) and $1 \mathrm{Mx}$ (KX375404.1), using DNAMAN v7 software [22]. This permitted the design of a primer pair (5'-CGCC CTCGTGGCTCTCACCC/5' -TTTGCTGCTGGTAT TGTCCA) which specifically amplified the encoding sequence of $1 \mathrm{Mx}$ subunit. The amplicon was generated by exposing the reaction to an initial denaturation of $94^{\circ} \mathrm{C} / 5$ min, followed by 30 cycles of $94^{\circ} \mathrm{C} / 30 \mathrm{~s}, 63^{\circ} \mathrm{C} / 30 \mathrm{~s}, 72^{\circ} \mathrm{C} /$ $40 \mathrm{~s}$; the PCR was completed with a final extension step of $72{ }^{\circ} \mathrm{C} / 10 \mathrm{~min}$.

\section{Results}

The FISH karyotype of ae. Geniculata

When the mitotic chromosomes of Ae. geniculata were probed with labeled total genomic DNA of Ae. comosa 
and an excess of unlabeled Ae. umbellulata genomic DNA, the M genome chromosomes (labeled green) were readily distinguished from the $U$ genome ones (Fig. 1a). The same preparations were then subjected to FISH using the four probes pSc119.2, Afa family repeats, pTa71 and $(\mathrm{CTT})_{5}$ following suggestions made elsewhere in the literature [10-12, 23, 24]. Most of the pSc119.2 sites were found to lie near the telomeres, while those recognized by the Afa family repeats probe were limited to chromosomes $2 \mathrm{M}^{\mathrm{g}}, 3 \mathrm{M}^{\mathrm{g}}$ and $7 \mathrm{M}^{\mathrm{g}}$ (Fig. 1b). The FISH profile of AS6 differed somewhat from previously published profiles of the species: for instance, pTa71 sites were located on seven pairs of chromosomes, of which two were on $U$ genome chromosomes $\left(1 U^{g}, 5 U^{g}\right)$ and the other five on the $M$ genome chromosomes $1 \mathrm{M}^{\mathrm{g}}$, $2 \mathrm{M}^{\mathrm{g}}, 3 \mathrm{M}^{\mathrm{g}}, 5 \mathrm{M}^{\mathrm{g}}$ and $6 \mathrm{M}^{\mathrm{g}}$ (Fig. 1b). The $(\mathrm{CTT})_{5}$ probe detected sites on the chromosomes belonging to both the $\mathrm{U}$ and the $\mathrm{M}$ genomes (Fig. 1c). A FISH karyotype of AS6 based on probing with pSc119.2, Afa family repeats, pTa71 and $(\mathrm{CTT})_{5}$ is shown in Fig. 1d. Without the GISH data, it was not easy to differentiate between several of the $\mathrm{M}$ and $\mathrm{U}$ genome chromosomes: for example, the FISH profiles of chromosomes $3 \mathrm{U}^{\mathrm{g}}$ and $4 \mathrm{M}^{\mathrm{g}}$ were almost identical to one another (Fig. 1d). For this reason, experiments were carried out to elaborate a more effective set of FISH probes; these led to the choice of the combination pTa-713, $(\mathrm{AAC})_{5}$ and pTa71. The $(\mathrm{ACC})_{5}$ sites were largely concentrated around the centromeres and the middle of the chromosome arms, while the pTa-713 sites were distributed across several chromosome arms (Fig. 1e, f). The three probes combination allowed for each of the 14 chromosomes of Ae. geniculata to be discriminated without the need for an accompanying GISH procedure (Fig. 1f).


Fig. 1 The FISH/GISH karyotype of Ae. geniculata accession AS6. a GISH differentiates the M genome chromosomes (labeled green) from those of the $\mathrm{U}$ genome (blue). b, c FISH profiling of the mitotic chromosomes of AS6 using as probes (b) pSc119.2 (red), Afa family repeats (green) and pTa71 (yellow), (c) (CTT) 5 (red). d The FISH karyotype of AS6. The left hand chromosome of each pair shows the hybridization sites of pSc119.2, Afa family repeats and pTa71, while the right hand chromosome of each pair shows the $(\mathrm{CTT})_{5}$ sites. e FISH profiling of the mitotic chromosomes of AS6 using as probes pTa-713 (red), (AAC) 5 (green) and pTa71 (yellow). f The FISH karyotype of AS6, based on pTa-713, (AAC) 5 and pTa71 sites. The images are shown in $(a, b, c)$ and (e) were obtained from a single mitotic cell 


\section{FISH-based identification of introgression materials}

Ten derivatives of a cross between Ae. geniculata and bread wheat were subjected to the newly developed FISH assay. One line had a somatic number of 44, while that of the nine others was 42 chromosomes. A GISH analysis demonstrated that two of the chromosomes present in the $2 \mathrm{n}=44$ line (Add L-1) had been inherited from Ae. geniculata (Fig. 2a). Applying the FISH procedure confirmed that these chromosomes comprised a pair of $1 \mathrm{M}^{\mathrm{g}}$ chromosomes (Fig. 2b, c). Three of the $2 \mathrm{n}=42$ lines also carried a pair of Ae. geniculata chromosomes (Fig. 2d), and the FISH assay showed that all three (Sub L-1, Sub L-2, and Sub L-3) represented a $4 \mathrm{M}^{\mathrm{g}}(4 \mathrm{~B})$ substitution line (Fig. 2e, f). There was no evidence for the presence of any Ae. geniculata chromatin in any of the other six lines.

\section{The HMW-GS profile of add L-1}

Since homeologous group 1 chromosomes harbor the Glu-1 genes which encode HMW-GS, the endosperm protein profile of Add L-1 grain was obtained by SDS-PAGE to identify the presence of the products of the Ae. geniculata homeolog of Glu-1. The Add L-1 and AS6 profiles both included a subunit not represented in either of the wheat parents of Add L-1 or in any of the other sister lines (Fig. 3). When the Glu-1 coding region was PCR-amplified from Ae. geniculata gDNA, the amplicon was found to include a fragment of the same length as that present in the amplicon generated from an Add L-1 template; the fragment was not represented in amplicons produced from euploid wheat (Fig. 4a). When this fragment was cloned and sequenced, both the Add L-1 and AS6 version proved to be a sequence of length $1860 \mathrm{nt}$ and were of identical sequence. This sequence has been deposited in Genbank under accession number MK135469. The sequence differed from that encoding the $1 \mathrm{My}$ subunit (KX375405.1) with respect to seven nucleotides, and their predicted products differed for just one residue. The amplified fragment derived from the gene encoding the AS6 $1 \mathrm{Mx}$ subunit was likely



Fig. 2 Identification of Ae. geniculata chromatin in derivatives of an Ae. geniculata $\times$ wheat wide cross. a-c A chromosome $1 \mathrm{M}^{9}$ disomic addition line was recognized following (a) GISH using labeled AS6 genomic DNA as the probe, (b, c) FISH with probes (b) pTa-713 and (AAC) 5, $^{(\mathbf{c})}$ pSc119.2 and pTa-535. The images shown in (a-c) were obtained from a single mitotic cell. $\mathbf{d}-\mathbf{f} A \mathbf{A M}^{\mathrm{g}}(4 \mathrm{~B})$ substitution line was recognized following (d) GISH using labeled AS6 genomic DNA as the probe, (e, f) FISH with probes (e) pTa-713 and (AAC), (f) pSc119.2 and pTa-535. The images shown in (d-f) were obtained from a single mitotic cell 




Fig. 3 HMW-GS profiling of the endosperm proteins of Add L-1. Lane 1: Chinese Spring, lane 2: AS6, lane 3: YY2, lane 4: CM41, lane 5, 6: an Ae. geniculata $\times$ wheat derivative lacking Ae. geniculata chromatin, lane 7: Add L-1. YY2 and CM41 are part of the pedigree of Add L-1. The arrow indicates the HMW-GS present in the endosperm protein of both Add L-1 and AS6

too similar to one of the wheat fragments (Fig. 4a) to facilitate its isolation, so a PCR assay was designed to target this gene in order to confirm its presence in Add L-1 (Fig. 4b). The conclusion drawn from these assays was that the Ae. geniculata chromosome present in Add L-1 harbored the genes encoding both the $\mathrm{x}$ and $\mathrm{y}$ subunits of Glu-M1.

\section{SNP genotyping of the $4 M^{9}(4 B)$ substitution lines}

The three $4 \mathrm{M}^{\mathrm{g}}(4 \mathrm{~B})$ substitution lines Sub L-1 through L-3, along with their Ae. geniculata parent AS6 and their wheat parents YY2 and CM41 were subjected to SNP genotyping to confirm their FISH-based designation. It was expected that the wheat SNP markers map to 4B (2601) showed a highest ratio to present as missing in the $4 \mathrm{M}^{\mathrm{g}}(4 \mathrm{~B})$ lines. Thus, the missing markers ratio for individual chromosomes were analyzed. As expected, about $60 \%$ SNPs $(59.0 \%$ for Sub L-1; $60.0 \%$ for Sub L-2; $60.6 \%$ for Sub L-3) for chromosome $4 \mathrm{~B}$ that covered the whole chromosome (Fig. 5) showed as missing in the three substitution lines and this ratio greatly exceeded for other chromosomes (Table 1).
Of the 51,159 features represented on the SNP chip, 29,537 (57.7\%) also hybridized with a sequence(s) present in AS6. Comparability, about 40\% 4B SNPs also present in the three $4 \mathrm{M}^{\mathrm{g}}(4 \mathrm{~B})$ substitution lines. It suggested a proportion of the SNP assays also recognized a site on $4 \mathrm{M}^{\mathrm{g}}$. To obtain these SNPs, we filtered 4B SNPs among Sub L-1, Sub L-2, Sub L-3 and AS6. However, ambiguous hybridizing signals may appear using wheat SNP markers to genotype its relative species and introgression lines [25]. Thus, only homozygous SNPs were considered. In all, 240 SNPs covering the whole 4B chromosome presenting in each of the three Sub L lines and AS6, and presenting as same homozygous alleles possibly shared by $4 \mathrm{M}^{\mathrm{g}}$ and $4 \mathrm{~B}$ were obtained (Additional file 1: Table S1).

\section{Discussion}

The ability to identify the extent, location and origin of chromatin introgressed into a crop species genome from a species in its tertiary genepool is important for the successful execution of a chromosome engineering experiment. FISH-based karyotyping based on a panel of multi-copy probes can be an effective means of recognizing individual chromosomes [11, 17, 26, 27]. Here, when a set of seven such probes was applied to derive a FISH-based karyotype of $A e$. geniculata, a combination of just three of them, namely pTa-713, (AAC) $)_{5}$ and pTa71, was sufficient to discriminate clearly between each of the species' 14 chromosomes. The FISH assay could then be applied to detect the origin of Ae. geniculata chromatin introgressed into a number of derivatives of a wide cross between this species and wheat, resulting in the recognition of a chromosome $1 \mathrm{M}^{\mathrm{g}}$ disomic addition line and three $4 \mathrm{M}^{\mathrm{g}}(4 \mathrm{~B})$ disomic substitution lines.

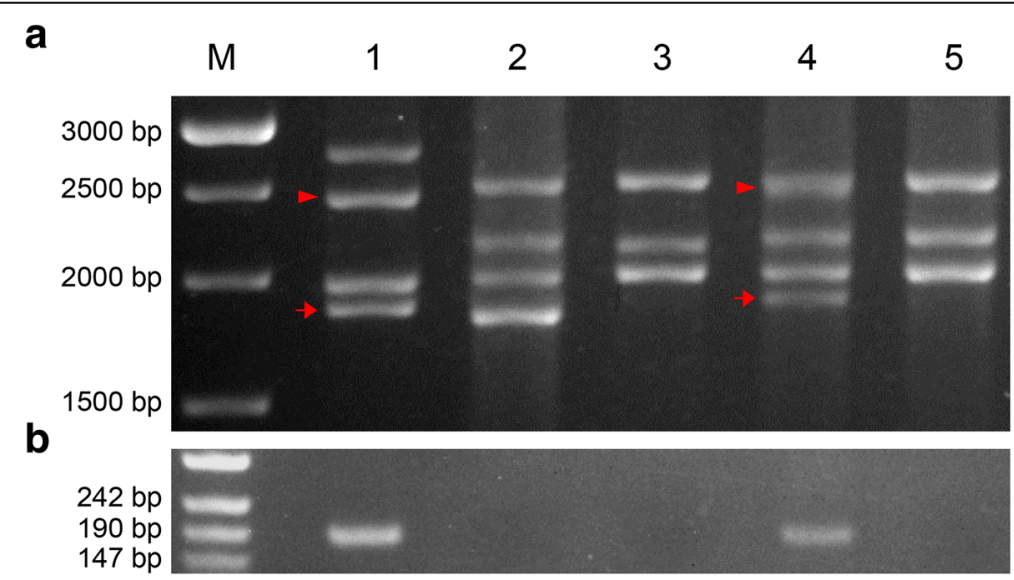

Fig. 4 PCR-based genotyping of Add L-1. Lane M: weight marker, lane 1: AS6, lane 2: YY2, lane 3: CM41, lane 4: Add L-1, lane 5: an Ae. geniculata $\times$ wheat derivative lacking Ae. geniculata chromatin. The amplicons were generated by a primer pair targeting (a) all Glu- 1 sequences, (b) the gene encoding the $x$ subunit of Glu-M1. The tailed arrow in (a) indicates the fragment amplified from the sequence encoding the Glu-M1y subunit and the tailless arrow from the sequence encoding the Glu-M1x subunit 




Fig. 5 The map location of the $4 B$ SNPs not present in the three $4 M^{9}(4 B)$ substitution lines Sub $L-1$ through $L-3$

High density SNP arrays have been shown to be effective for the genotyping of wheat and certain wheat/alien introgression lines $[25,28]$. In species containing genomes closely related to those present in wheat, the expectation is that - depending on the extent of the evolutionary separation involved - a proportion of the SNP assays developed within wheat itself will also detect the presence of alien chromatin. Here, around $60 \%$ of the wheat SNP loci recognized a site in the AS6 genome, a consequence of the fairly close relationship between Ae. geniculata and bread wheat [29-31]. A set of 240 of the 2601 chromosome 4B SNPs behaved in this manner, highlighting loci on both chromosome $4 \mathrm{~B}$ and chromosome $4 \mathrm{M}^{\mathrm{g}}$ (Additional file 1: Table S1). The conclusion

Table 1 The distribution of missing SNPs on chromosomes in substitution lines

\begin{tabular}{|c|c|c|c|c|c|c|c|}
\hline \multirow[t]{2}{*}{ Chromosome } & \multirow[t]{2}{*}{ Number } & \multicolumn{2}{|l|}{ Sub L1 } & \multicolumn{2}{|l|}{ Sub L2 } & \multicolumn{2}{|l|}{ Sub L3 } \\
\hline & & Number & Ratio & Number & Ratio & Number & Ratio \\
\hline $1 \mathrm{~A}$ & 2632 & 35 & $1.3 \%$ & 28 & $1.1 \%$ & 26 & $1.0 \%$ \\
\hline $1 \mathrm{~B}$ & 2630 & 27 & $1.0 \%$ & 36 & $1.4 \%$ & 27 & $1.0 \%$ \\
\hline $1 \mathrm{D}$ & 2495 & 19 & $0.8 \%$ & 24 & $1.0 \%$ & 21 & $0.8 \%$ \\
\hline $2 \mathrm{~A}$ & 2622 & 14 & $0.5 \%$ & 18 & $0.7 \%$ & 20 & $0.8 \%$ \\
\hline $2 \mathrm{~B}$ & 2578 & 33 & $1.3 \%$ & 31 & $1.2 \%$ & 45 & $1.7 \%$ \\
\hline $2 \mathrm{D}$ & 2590 & 204 & $7.9 \%$ & 207 & $8.0 \%$ & 205 & $7.9 \%$ \\
\hline $3 A$ & 2194 & 31 & $1.4 \%$ & 24 & $1.1 \%$ & 32 & $1.5 \%$ \\
\hline $3 B$ & 2629 & 32 & $1.2 \%$ & 34 & $1.3 \%$ & 46 & $1.7 \%$ \\
\hline $3 D$ & 2072 & 17 & $0.8 \%$ & 21 & $1.0 \%$ & 23 & $1.1 \%$ \\
\hline $4 \mathrm{~A}$ & 2573 & 34 & $1.3 \%$ & 35 & $1.4 \%$ & 41 & $1.6 \%$ \\
\hline $4 B$ & 2601 & 1535 & $59.0 \%$ & 1560 & $60.0 \%$ & 1575 & $60.6 \%$ \\
\hline $4 \mathrm{D}$ & 1087 & 28 & $2.6 \%$ & 36 & $3.3 \%$ & 30 & $2.8 \%$ \\
\hline $5 A$ & 2633 & 37 & $1.4 \%$ & 53 & $2.0 \%$ & 44 & $1.7 \%$ \\
\hline $5 B$ & 2622 & 39 & $1.5 \%$ & 50 & $1.9 \%$ & 64 & $2.4 \%$ \\
\hline $5 D$ & 2142 & 33 & $1.5 \%$ & 40 & $1.9 \%$ & 48 & $2.2 \%$ \\
\hline $6 \mathrm{~A}$ & 2623 & 40 & $1.5 \%$ & 49 & $1.9 \%$ & 40 & $1.5 \%$ \\
\hline $6 \mathrm{~B}$ & 2601 & 94 & $3.6 \%$ & 107 & $4.1 \%$ & 91 & $3.5 \%$ \\
\hline $6 \mathrm{D}$ & 2067 & 30 & $1.5 \%$ & 30 & $1.5 \%$ & 24 & $1.2 \%$ \\
\hline $7 \mathrm{~A}$ & 2601 & 28 & $1.1 \%$ & 42 & $1.6 \%$ & 41 & $1.6 \%$ \\
\hline $7 B$ & 2542 & 212 & $8.3 \%$ & 219 & $8.6 \%$ & 198 & $7.8 \%$ \\
\hline $7 D$ & 2625 & 23 & $0.9 \%$ & 23 & $0.9 \%$ & 23 & $0.9 \%$ \\
\hline
\end{tabular}


was that a wheat SNP array can be highly informative for identifying the presence of a wheat/alien chromosome substitution line.

The derivatives of the Ae. geniculate $\times$ wheat wide cross have inherited their non-nuclear genomes from Ae. geniculata, which has resulted in their exhibiting very late maturity [32]. While this trait has complicated the evaluation of their agronomic performance, it is of some interest that the presence of chromosome $4 \mathrm{M}^{\mathrm{g}}$ induces the formation of supernumerary florets, and also introduces a gametocidal mechanism which generates chromosome breakage in gametes which lack the chromosome [33, 34]. The product of Glu-M1 has been associated with a positive effect on the rheological strength of doughs made from flour of grains carrying chromosome $1 \mathrm{M}^{\mathrm{g}}[8,9]$. Thus, both the $1 \mathrm{M}^{\mathrm{g}}$ addition line and the $4 \mathrm{M}^{\mathrm{g}}(4 \mathrm{~B})$ substitution lines can provide the starting material for producing alien translocation lines of interest to the wheat breeding community.

\section{Conclusion}

This study has established a FISH protocol able to unequivocally identify each of the 14 chromosomes of $A e$. geniculata, avoiding the need to include a parallel GISH procedure. A wheat SNP array was successfully deployed to confirm the cytogenetic status of three independent $4 \mathrm{M}^{\mathrm{g}}(4 \mathrm{~B})$ substitution lines. Both the $1 \mathrm{M}^{\mathrm{g}}$ addition line and the $4 \mathrm{M}^{\mathrm{g}}(4 \mathrm{~B})$ substitution lines represent materials of potential utility for wheat improvement.

\section{Additional file}

Additional file 1: Table S1. The 240 SNP markers shared by Sub L-1, Sub L-2, Sub L-3 and AS6. (XLSX $5838 \mathrm{~kb}$ )

\section{Acknowledgements}

We thank the anonymous reviewers of the manuscript for their useful comments.

\section{Funding}

This research was financially supported by the Chinese government's National Key Research and Development Program (2016YFD0102000), the Sichuan Provincial Agricultural Department's Innovative Research Team (wheat-10), and the Sichuan Province's Science \& Technology Department Crops Breeding Project (2016NYZ0030)

\section{Availability of data and materials}

The raw SNP data and germplasm are available upon request to Shunzong Ning, Triticeae Research Institute, Sichuan Agricultural University, (email address:ningshunzong@126.com .).

\section{Authors' contributions}

YJY, KZ, MH, SZN, LBZ, and DCL designed the study, YJY, KZ, LBZ and KX conducted the experiments, YJY, KZ, SZN, LBZ, MH, LQZ, ZWY and DCL analyzed the results and $Y J Y, K Z, M H, S Z N$, and DCL wrote the manuscript. All the authors have read and approved the final version of the manuscript.

Ethics approval and consent to participate Not applicable.
Consent for publication

Not applicable.

\section{Competing interests}

The authors declare that they have no competing interests.

\section{Publisher's note}

Springer Nature remains neutral with regard to jurisdictional claims in published maps and institutional affiliations.

Received: 24 December 2018 Accepted: 26 March 2019

Published online: 02 April 2019

References

1. Kimber G, Sallee PJ, Feiner MM. The interspecific and evolutionary relationships of Triticum ovatum. Genome. 1988;30(2):218-21.

2. Kuraparthy V, Chhuneja P, Dhaliwal HS, Kaur S, Bowden RL, Gill BS. Characterization and mapping of cryptic introgression from Ae geniculata with new leaf rust and stripe rust resistance genes $L r 57$ and $Y_{r} 40$ in wheat. Theor Appl Genet. 2007;114(8):1379-89.

3. Liu WX, Rouse M, Friebe B, Jin Y, Gill B, Pumphrey MO. Discovery and molecular mapping of a new gene conferring resistance to stem rust, Sr53, derived from Aegilops geniculata, and characterization of spontaneous translocation stocks with reduced alien chromatin. Chromosom Res. 2011; 19(5):669-82.

4. Friebe B, Heun M. C-banding pattern and powdery mildew resistance of Triticum ovatum and four T. aestivum-T. ovatum chromosome addition lines. Theor Appl Genet. 1989;78(3):417-24.

5. Zeller FJ, Konig L, Hartl L, Mohler V, Hsam SLK. Chromosomal location of genes for resistance to powdery mildew in common wheat (Triticum aestivum L. em Thell.) 7. Gene Pm29 in line Pova. Euphytica. 2002;123(2): 187-94.

6. Zaharieva M, Gaulin E, Havaux M, Acevedo E, Monneveux P. Drought and heat responses in the wild wheat relative Aegilops geniculata Roth: potential interest for wheat improvement. Crop Sci. 2001:41:1321-9.

7. Pradhan GP, Prasad PW, Fritz AK, Kirkham MB, Gill BS. Response of Aegilops species to drought stress during reproductive stages of development. Funct Plant Biol. 2011;39(1):51-9.

8. Grag M, Tsujimoto H, Gupta RK, Kumar A, Kaur N, Kumar R, et al. Chromosome specific substitution lines of Aegilops geniculata alter parameters of bread making quality of wheat. PLoS One. 2016;11(10): e0162350.

9. Alvarez JB, Guzmán C. Interspecific and intergeneric hybridization as a source of variation for wheat grain quality improvement. Theor Appl Genet. 2018;131(2):225-51.

10. Molnár I, Cifuentes M, Schneider A, Benavente E, Molnár-Láng M. Association between simple sequence repeat-rich chromosome regions and intergenomic translocation breakpoints in natural populations of allopolyploid wild wheats. Ann Bot. 2011;107(1):65-76.

11. Kwiatek M, Wiśniewska H, Apolinarska B. Cytogenetic analysis of Aegilops chromosomes, potentially usable in triticale ( $\times$ Triticosecale Witt.) breeding. J Appl Genet. 2013;54:147-55.

12. Molnár I, Kubaláková M, Šimková H, Cseh A, Molnár-Láng M, Doležel J. Chromosome isolation by flow sorting in Aegilops umbellulata and $\mathrm{Ae}$. comosa and their allotetraploid hybrids Ae. biuncialis and Ae. geniculata. PLoS One. 2011;6(11):e27708.

13. Zhao LB, Ning SZ, Yu JJ, Hao M, Zhang LQ, Yuan ZW, et al. Cytological identification of an Aegilops variabilis chromosome carrying stripe rust resistance in wheat. Breeding Sci. 2016;66:522-9.

14. Hao M, Luo JT, Yang M, Zhang LQ, Yan ZH, Yuan ZW, et al. Comparison of homoeologous chromosome pairing between hybrids of wheat genotypes Chinese spring ph1b and Kaixian-luohanmai with rye. Genome. 2011;54(12): 959-64.

15. Zhao LB, Ning SZ, Yi YJ, Zhang LQ, Yuan ZW, Wang JR, et al. Fluorescence in situ hybridization karyotyping reveals the presence of two distinct genomes in the taxon Aegilops tauschii. BMC Genomics. 2018;19(1):3.

16. Nagaki K, Tsujimoto H, Isono K, Sasakuma T. Molecular characterization of a tandem repeat, Afa family, and its distribution among Triticeae. Genome. 1995;38(3):479-86. 
17. Tang ZX, Yang ZJ, Fu SL. Oligonucleotides replacing the roles of repetitive sequences pAs1, pSc1 19.2, pTa-535, pTa71, CCS1, and pAWRC.1 for FISH analysis. J Appl Genet. 2014;55(3):313-8.

18. Cuadrado A, Schwarzacher T, Jouve N. Identification of different chromatin classes in wheat using in situ hybridization with simple sequence repeat oligonucleotides. Theor Appl Genet. 2000;101(5-6):711-7.

19. Wickham H. ggplot2: elegant graphics for data analysis. New York: SpringerVerlag; 2016.

20. Yan ZH, Wan YF, Liu KF, Zheng YL, Wang DW. Identification of a novel HMW glutenin subunit and comparison of its amino acid sequence with those of homologous subunits. Chin Sci Bull. 2002:47(3):222-6.

21. Guo XH, Wu BH, Hu XG, Bi ZG, Wang ZZ, Liu DC, et al. Molecular characterization of two y-type high molecular weight glutenin subunit alleles 1 Ay $12^{*}$ and 1 Ay $8^{*}$ from cultivated einkorn wheat (Triticum monococcum ssp. monococcum). Gene. 2013:516(1):1-7.

22. Wei $L$, Wang $Q$, Zhang $L L, M a ~ J$, Wang JR, Qi PF, et al. Genetic analyses of Glu $-15^{\text {sh }}$ in wheat/Aegilops sharonensis hybrid progenies and development of alien HMW-GSs gene-specific markers. Mol Breeding. 2015;35(12):230.

23. Badaeva ED, Amosova AV, Samatadze TE, Zoshchuk SA, Shostak NG, Chikida $\mathrm{NN}$, et al. Genome differentiation in Aegilops. 4. Evolution of the U-genome cluster. Plant Syst Evol. 2004;246(1/2):45-76.

24. Landjeva S, Kocheva K, Karceva T, Sepsi A, Molnár I, Schneider A, et al. Molecular cytogenetic identification of a wheat - Aegilops geniculata Roth spontaneous chromosome substitution and its effects on the growth and physiological responses of seedlings to osmotic stress. Plant Breed. 2012; 131(1):81-7.

25. Zhou SH, Zhang JP, Che YH, Liu WH, Lu YQ, Yang XM, et al. Construction of Agropyron Gaertn. Genetic linkage maps using a wheat 660K SNP array reveals a homoeologous relationship with the wheat genome. Plant Biotechnol J. 2017;16:818.

26. Pedersen C, Langridge P. Identification of the entire chromosome complement of bread wheat by two-colour FISH. Genome. 1997;40(5):589-93.

27. Komuro S, Endo R, Shikata K, Kato A. Genomic and chromosomal distribution patterns of various repeated DNA sequences in wheat revealed by a fluorescence in situ hybridization procedure. Genome. 2013;56(3):131-7.

28. Winfield MO, Allen AM, Burridge AJ, Baker GL, Benlow HR, Wikinson PA, et al. High-density SNP genotyping array for hexaploid wheat and its secondary and tertiary gene pool. Plant Biotechnol J. 2016;14(5):1195-206.

29. Tiwari VK, Wang SC, Sehgl S, Vrána J, Friebe B, Kubaláková M, et al. SNP discovery for mapping alien introgressions in wheat. BMC Genomics. 2014; 15(1):273.

30. Tiwari VK, Wang SC, Danilova T, Koo D, Vrána j KM, et al. Exploring the tertiary gene pool of bread wheat: sequence assembly and analysis of chromosome $5 \mathrm{M}^{9}$ of Aegilops geniculata. Plant J. 2016;84(4):733-46.

31. Molnár I, Vrána J, Burešová V, Cápal P, Farkas A, Darkó É, et al. Dissecting the U, M, S and C genomes of wild relatives of bread wheat (Aegilops spp.) into chromosomes and exploring their synteny with wheat. Plant J. 2016;88(3): 452-67.

32. Wu YW, Zhang CL, Liu CG, Ren SX, Zhang Y. Breeding technology of alloplasmic wheat. Sci China Ser C Life Sci. 1998;41(5):449-58.

33. Friebe BR, Tuleen NA, Gill BS. Development and identification of a complete set of Triticum aestivum-Aegilops geniculata, chromosome addition lines. Genome. 1999:42(3):374-80.

34. Kwiatek MT, Wiśniewska H, Ślusarkiewicz-Jarzina A, Majka J, Majka M, Belter J, et al. Gametocidal factor transferred from Aegilops geniculata Roth can be adapted for large-scale chromosome manipulations in cereals. Front Plant Sci. 2017;8:409.

\section{Ready to submit your research? Choose BMC and benefit from:}

- fast, convenient online submission

- thorough peer review by experienced researchers in your field

- rapid publication on acceptance

- support for research data, including large and complex data types

- gold Open Access which fosters wider collaboration and increased citations

- maximum visibility for your research: over $100 \mathrm{M}$ website views per year

At BMC, research is always in progress.

Learn more biomedcentral.com/submissions 